\title{
Reconstruction with Parallel Projections using ART
}

\author{
Nirvikar \\ Shobhit University \\ Meerut, India
}

\author{
Raghuvir Singh \\ Shobhit University \\ Meerut, India
}

\begin{abstract}
Algebraic Reconstruction Technique (ART) is most accurate method for image reconstruction. In present paper the accuracy of ART is shown with parallel projections, in all only 16 projections with about 60 iterations are used to obtain reconstruction.

\section{Keywords}

ART, CT, Image processing, Projections, Reconstruction
\end{abstract}

\section{INRODUCTION}

The word tomography means "reconstruction from projections", i.e. the recovery of a function from its line or (hyper) plane integrals (from the Greek - slice and -to write). In the applied sense, it is a method to reconstruct cross sections of the interior structure of an object without destructing or damage the object. The term often occurs in the combination computerized (computed) tomography (CT) or computer-assisted tomography (CAT), since for performing the reconstruction in practice one needs the use of a digital computer. The initial use of computed tomography (CT) for applications in radiological diagnostics during the seventies sparked a revolution in the field of medical engineering [3][7]. More recently, however, medical imaging has also been successfully accomplished with radioisotopes, ultrasound, and magnetic resonance. There are numerous nonmedical imaging application, which lend themselves to the methods of CT like the mapping of underground resources via crossboreholes imaging (e.g. estimation of depth to water table, geological and hydrological mapping etc.), some specialized cases of cross sectional imaging for nondestructive testing, the determination of the brightness distribution over a celestial sphere, and three dimensional imaging with electron microscopy. And even throughout the eighties, a CT examination lost little if any of its special and exclusive character. In the meantime, however, times have changed. Today computed tomography represents a perfectly natural and established technology which has advanced to become an indispensable and integral component of routine work in clinics and medical practices.

The classic application of industrial X-ray computed tomography (CT) is the inspection and three-dimensional measurement of metal and plastic castings. However, phoenix x-ray's highresolution X-ray technology opens up a variety of new applications in fields such as sensor technology, electronics, materials science, and many other natural sciences.

In last years, industrial computed tomography (CT) in Switzerland had its main application in scientific examinations. Specific fields of interest were flaw detection, analysis of failure, dimensional measurements of not accessible geometrical features, inspection of assemblies or statistical investigations of material properties as density distribution. Single slices were taken at well-defined places and used for further analysis[4].

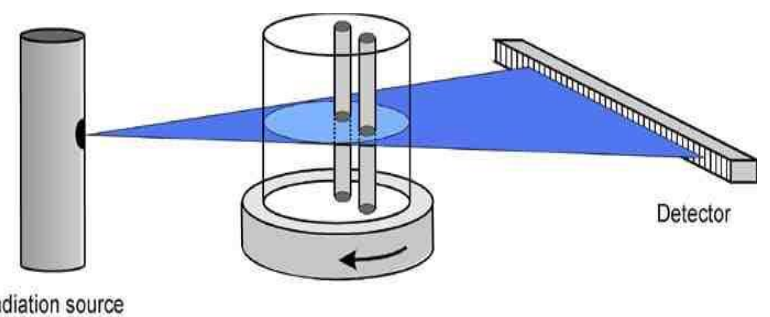

Fig 1. Computed Tomography Scan Process

Today, the most important application of CT has become scanning for 3D-digitizing purposes [5]. First of all, automotive and motorcycle industries as well as their suppliers and the medical technology show a very strong interest in the new possibilities that CT offers. Using this new technology it is possible to reduce the time to market for development of new products. Thus companies can realize substantial competitive advantages.

\section{ALGEBRAIC RECONSTRUCTION TECHNIQUE (ART)}

The Algebraic Reconstruction Technique (ART) introduced by Gordan, Bender and Herman[1] uses a large number of projections to reconstruct the 2-dimensional beam density distribution. Algebraic Reconstruction Techniques (ART) was first published in the biomedical imaging literature in 1970 [2]. From the mathematical point of view, they are variations of the iterative method for solving a system of simultaneous equations introduced by Kaczmarz in 1937 [3]. ART can produce highquality reconstructions with excellent computational efficiency.

The ART algorithms have a simple intuitive basis. Each projected density is thrown back across the reconstruction space in which the densities are iteratively modified to bring each reconstructed projection into agreement with the measured projection. Assuming that the object being reconstructed is enclosed in a square space of $\mathrm{n} \times \mathrm{n}$ array of small pixels, $p_{\mathrm{j}}\left(j=1 \ldots \ldots n^{2}\right)$ is grayness or density number, which is uniform within the pixel but different from other pixels. A "ray" is a region of the square space which lies between two parallel lines. The weighted ray sum is the total grayness of the reconstruction figure within the ray. The projection at a given angle is then the sum of non-overlapping, equally wide rays covering the object. The ART algorithm consists of altering the grayness of each pixel intersected by the ray in such a way as to make the ray sum agree with the corresponding element of the measured projection. 
The ART algorithm begins with some initial estimate of the image to be reconstructed (usually taken as a uniformly gray image). It modifies this estimate repeatedly until the pixel values appear to converge by some criterion. ART decides how to modify the image by summing the pixels along some straight path and comparing this sum to the measured ray sum (referred to earlier as an "X-ray projection"). The difference between projections calculated from the image estimate, and the measured ray is calculated, and the adjustment is divided among the pixels in the ray sum.

The methods of algebraic reconstruction techniques (ART) in computerized tomography are based on a representation of the projection line integrals as discrete ray-sums [2][6][7]. Let $p_{i}$ be the ray-sum measured with the $i$ th ray then the relationship between the $f_{\mathrm{j}}$ 's and $p_{\mathrm{i}}$ 's may be expressed as

$P_{i}=\sum_{j=1}^{N} w_{i j} f_{j}, i=1,2, \ldots, M$

where $M$ is the total number of rays(in all the projections) and $w_{i j}$ is the weighting factor that represents the contribution of the $j$ th cell to the $i$ th ray integral. The subscript $m$ represents the projection index from a total of $\boldsymbol{M}$ projections. The subscript $n$ represents the ray index among $N$ rays within each projection.

For large values of $\mathrm{M}$ and $\mathrm{N}$ there exist very attractive iterative methods for solving (1). These are based on the "method of projections" as first proposed by Kaczmarz [3], and later elucidated further by Tanabe [8]. To explain the computational steps involved in these methods, we first write (1) in an expanded form:

$$
\begin{gathered}
w_{11} f_{1}+w_{12} f_{2}+\cdots+w_{1 N} f_{N}=p_{1} \\
w_{21} f_{1}+w_{22} f_{2}+\cdots+w_{2 N} f_{N}=p_{2} \\
\cdot \\
w_{M 1} f_{1}+w_{M 2} f_{2}+\cdots+w_{M N} f_{N}=p_{M}
\end{gathered}
$$

Therefore, an image, represented by $\left(f_{1}, f_{2}, \ldots, f_{N}\right)$, may be considered to be a single point in an N-dimensional space. In this space each of the above equations represents a hyperplane. When a unique solution to these equations exists, the intersection of all these hyperplanes is a single point giving that solution.

The computational procedure for locating the solution consists of first starting with an initial guess, projecting this initial guess on the first line, reprojecting the resulting point on the second line, and then projecting back onto the first line, and so forth. If a unique solution exists, the iterations will always converge to that point.

For the computer implementation of this method, we first make an initial guess at the solution. This guess, denoted by $f_{1}^{(0)} f_{2}^{(0)}, x_{N} f_{N}^{(0)}$ is represented vectorially by $\vec{f}^{(0)}$ in the $\mathrm{N}$-dimensional space. In most cases, we simply assign a value of zero to all the $\mathrm{f}_{i}$ 's. This initial guess is projected on the hyperplane represented by the first equation in (2).

As mentioned before, the computational procedure for algebraic reconstruction consists of starting with an initial guess for the solution, taking successive projections on the hyperplanes represented by the equations in (2), eventually yielding $\vec{f}^{(M)}$. In the next iteration, $\vec{f}^{(M)}$ is projected on the hyperplane represented by the first equation in (2), and then successively onto the rest of the hyperplanes in (2), to yield $\vec{f}^{(2 M)}$, and so on. Tanabe [8] has shown that if there exists a unique solution $\vec{f}_{s}$ to the system of equations (2), then

$\lim _{k \rightarrow \infty} \vec{f}(k M)=\vec{f}$.

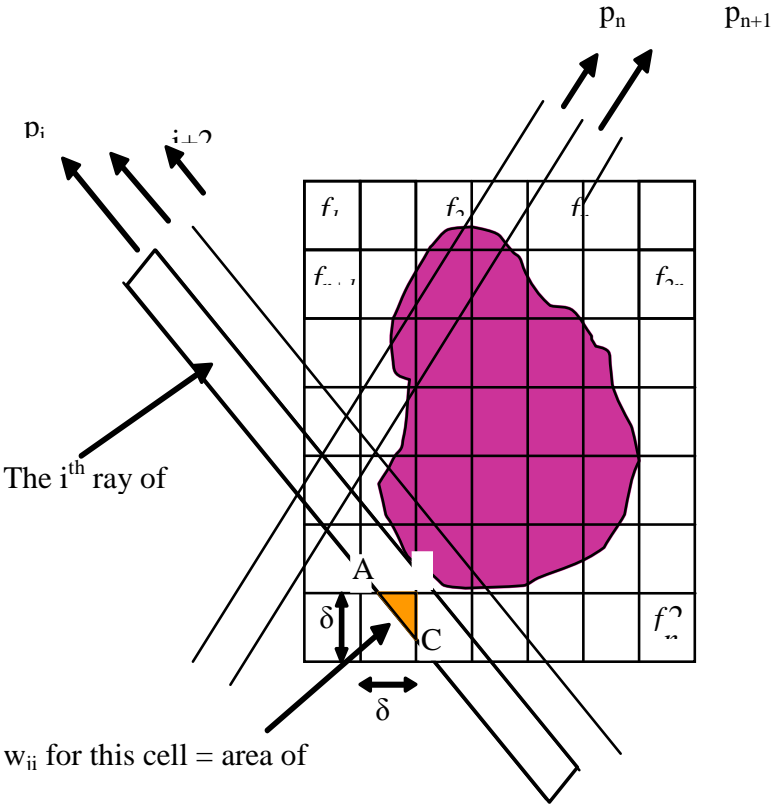

Fig 2. Image with a grid superimposed onto it, where image values are assumed to be constant within a cell.

When we project the $(i-1)$ th solution onto the $i$ th hyperplane [ $i$ th equation in (1)] the gray level of the $j$ th element, whose current value is $f_{j}^{(i-1)}$, is obtained by correcting its current value by $\Delta f_{j}^{(d)}$, where

$\Delta f_{j}^{(i)}=f_{j}^{(i)}-f_{j}^{(i-1)}=\frac{p_{i}-q_{i}}{\sum_{k=1}^{N} w_{i k}^{2}} w_{i j^{x}}$

where $\mathrm{p}_{i}$ is the measured ray-sum along the $i$ th ray, $\mathrm{q}_{i}$ is the computed ray-sum for the same ray based on the $(i-1)$ th solution for the image gray levels. The correction $\Delta f_{j}$, to the $j$ th cell is obtained by first calculating the difference between the measured ray-sum and the computed ray-sum, normalizing this difference by $\sum_{k=1}^{W} W_{i k^{3}}^{2}$ and then assigning this value to all the image cells in the $i$ th ray, each assignment being weighted by the corresponding $w_{i j}$. 
Generic ART procedure:

1. Prepare an initial estimate

2. Compute projections based on the guess

3. Refine the guess on the weighted difference between the actual projections and desired projections

4. Perform Steps 2 and 3 for all rays available

5. Repeat steps $2-4$ as many times as required

Different variations of the model can be used to determine the weight wij that each pixel $j$ contributes to the $i$ th weighted attenuation sum $\mathrm{P} i$. Let us use a model where each weight wij is the product of the pixel's attenuation $f j$ and the length of the ray's intersection with the pixel (expressed in pixel widths). The weights can then be determined geometrically from the angle and position of the ray (these are determined from the geometry of the scanner) and the chosen pixel dimensions.

ART simple example,

we have an image of $\mathrm{N}=9$ pixels. There are 3 detectors in the detector array, and the array is rotated through 4 views (horizontal, vertical, diagonal and antidiagonal) to produce $M=16$ raysums.

- make initial guess

- while convergence not reached //

- iteration for each projection

- for each ray

- compute back-projection

- compute difference to measured projection

- distribute difference

- end for

- end for

- end while

Starting with initial guess $f^{(0)}$ and projections $p$.

Table 1. Initial Image Data $\left(f^{(0)}\right)$

\begin{tabular}{|l|l|l|}
\hline 0 & 0 & 0 \\
\hline 0 & 0 & 0 \\
\hline 0 & 0 & 0 \\
\hline
\end{tabular}

Table 2. Initial Projection Value $(p)$

\begin{tabular}{|l|l|l|l|}
\hline 2 & 6 & 8 & 7 \\
\hline 9 & 7 & 2 & 1 \\
\hline 3 & 9 & 2 & 6 \\
\hline 8 & 7 & 9 & 7 \\
\hline
\end{tabular}

After 4 iteration

Table 3. Final Reconstructed Image

\begin{tabular}{|l|l|l|}
\hline 2.74 & 5.97 & 1.65 \\
\hline 2.47 & 2.59 & 5.68 \\
\hline 4.71 & 3.25 & 3.96 \\
\hline
\end{tabular}

Table 4. Projection data of final reconstructed image

\begin{tabular}{|l|l|l|l|}
\hline 10.36 & 10.74 & 11.92 & 9.92 \\
\hline 11.81 & 11.29 & 4.71 & 5.72 \\
\hline 9.29 & 11.65 & 1.65 & 2.74 \\
\hline 8.44 & 8.95 & 8.93 & 3.96 \\
\hline
\end{tabular}

Table 5. Error calculated in Image pixel values for every iteration

\begin{tabular}{|l|l|l|}
\hline Iteration & $\left|f_{i+1}-f_{i}\right|$ & $\left(f_{i+1}-f_{i}\right)^{2}$ \\
\hline 1 & 33.9999 & 143.9506 \\
\hline 2 & 4.6913 & 3.4226 \\
\hline 3 & 1.6872 & 0.5149 \\
\hline 4 & 0.8254 & 0.1038 \\
\hline
\end{tabular}

We are now done; because in this example the image has only nine pixels, it is convenient to present it as a line graph.

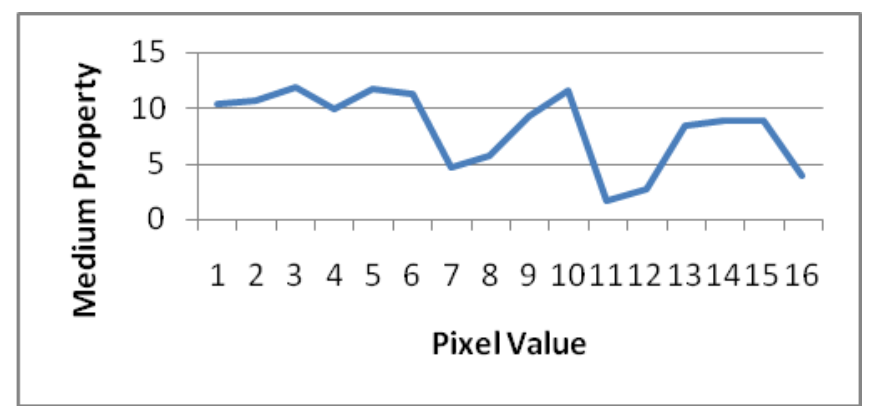

Fig 3. Image as a line Graph using ART with 4 iteration

In general, $M$ and $N$ are quite large. For example, when reconstructing an image size of $256 \times 256$ pixels, from 256 detector measurements in each of 256 views, $N$ and $M$ are both 65,536 . In such cases the weight matrix size is $65,536 \times 65,536=$ $4,294,967,296$. We require algorithms that are efficient in terms of both time and memory requirements to solve this on a computer without increasing turnaround time in the CT suite.

\section{CONCLUSION}

We have tested the algorithm on 3 X 3 image in which, we obtained four reconstructions iterative where errors in successive iterations are reducing very fast as it is evident from table 5. The algorithm now experimented with more numbers of projections to find the image data.

\section{REFERENCES}

[1] R. Gordon et al., "Three-Dimensional Reconstruction from Projections: A Review of Algorithms", International Review of Cytology, Vol. 38, p. 111 (1974).

[2] R. Gordon, R. Bender, and G. T. Herman, "Algebraic reconstruction techniques (ART) for three-dimensional 
electron microscopy and X-ray photography,” J. Theoret. Biol., vol. 29, pp. 471-482, 1970.

[3] F.Natterer, " The Mathematics of Computed Tomography", John Wiley and Sons, New York, 1986.

[4] A.H. Andersen, "Algebraic Reconstruction in CT from Limited Views" , IEEE Trans. On Medical Imaging, Vol. 8, pp 50-55, 1989.

[5] S. Kaczmarz, "Angentihrte Auflosung von Systemen linearer Gleichungen," Bull. Int. Acad. Pol. Sei. Lett., A, vol. 35, pp. 355-357, 1937.

[6] Flisch et al, ETH Zürich "Industrial Computed Tomography in Reverse Engineering Applications" Industrial Applications and Image Processing in Radiology March, 15 - 17, 1999 Berlin, Germany.

[7] J. Friedhoff, Aufbereitung von 3D-Digitalisierdaten für den Werkzeug-, Formen-und Modellbau, Vulkan Verlag, Essen 1997.
[8] Herman, G.T., Image Reconstruction from Projections: The Fundamentals of Compuerized Tomography, Academic Press, New York, 1980.

[9] C. N. Hounsfeld. "A method of and apparatus for examination of a body by radiation such as or Gamma radiation." Patent Specification 1283915, London, 1968.

[10] K. Tanabe, "Projection method for solving a singular system,” Numer. Math., vol. 17, pp. 03-214, 1971.

[11] A. H. Andersen and A. C. Kak, "Simultaneous algebraic reconstruction technique (SART): A superior implementation of the art algorithm," Ultrason. Imaging, vol. 6, pp. 81-94, Jan. 1984.

[12] Tanuja Srivastava, Raghuveer Singh, Nirvikar, “ ART with 3 projections in CT", CICON - 2010, pp. 1-3, May $8-9,2010$. 